\title{
A flexible approach to location problems
}

\author{
Antonio M. Rodriguez-Chia * Stefan Nickel ${ }^{\dagger} \quad$ Justo Puerto ${ }^{\ddagger}$ \\ Francisco R. Fernandez ${ }^{\ddagger}$
}

November 25, 1997

\begin{abstract}
In continuous location problems we are given a set of existing facilities and we are looking for the location of one or several new facilities. In the classical approaches weights are assigned to existing facilities expressing the importance of the new facilities for the existing ones.

In this paper, we consider a pointwise defined objective function where the weights are assigned to the existing facilities depending on the location of the new facility. This approach is shown to be a generalization of the median, center and centdian objective functions. In addition, this approach allows to formulate completely new location models. Efficient algorithms as well as structural results for this algebraic approach for location problems are presented. A complexity analysis and extensions to the multifacility and restricted case are also considered.
\end{abstract}

Keywords:Location Theory, Global optimization, Algebraic optimization, Convexity.

\section{Introduction}

In the last three decades a lot of research has been done in the field of continuous location theory and very much different models have been developed. For a comprehensive overview the reader is referred to Plastria's chapter in the book of Drezner [18].

In the following we will introduce a diferent model for location problems. This model provides a common framework for the classical continuous location problems and allows an algebraic approach to these problems. Moreover, this flexible apporach also leads to completely new objective functions for location problems.

We are given a gauge $\gamma(\cdot): \mathbb{R}^{n} \longrightarrow \mathbb{R}$ to measure distances, a set of demand points $A:=\left\{a_{1}, a_{2}, \ldots, a_{M}\right\} \subset \mathbb{R}^{n}$ (representing existing facilities or clients) and two

*Facultad de Ciencias del Mar. Universidad de Cadiz. Poligono Rio San Pedro. Puerto Real, Cadiz, Spain.

${ }^{\dagger}$ Fachbereich Mathematik. Universitat Kaiserslautern. Germany.

${ }^{\ddagger}$ Facultad de Matematicas. Universidad de Sevilla. C/Tarfia s/n, 41012 Sevilla, Spain. To whom all correspondence should be addressed. 
sets of non negative scalars $W=\left\{w_{1}, \ldots, w_{M}\right\}$ and $\Lambda=\left\{\lambda_{1}, \ldots, \lambda_{M}\right\}$. The element $w_{i} \in W$ is the weight of importance given to the existing facility $a_{i}$ and the elements of $\Lambda$ allows to choose between different kinds of objective functions.

Given a permutation $\sigma$ of the set $\{1, \ldots, M\}$ verifying

$$
w_{\sigma_{1}} \gamma\left(x-a_{\sigma_{1}}\right) \leq w_{\sigma_{2}} \gamma\left(x-a_{\sigma_{2}}\right) \leq \ldots \leq w_{\sigma_{M}} \gamma\left(x-a_{\sigma_{M}}\right)
$$

we denote $\gamma(x-A)_{(i)}=w_{\sigma_{i}} \gamma\left(x-a_{\sigma_{i}}\right)$.

The ordered Weber problem is then given by:

$$
\min _{x \in \mathbb{R}^{n}} F(x)=\sum_{i=1}^{M} \lambda_{i} \gamma(x-A)_{(i)} .
$$

Note that the problem is well-defined even if ties occur. In that case any order of the tied positions gives the same value.

Theoretical properties of (1) have been studied in a different setting in [19]. In the following we will refer to this problem as $1 / \mathbb{R}^{n} / \bullet / \gamma_{B} / \sum_{\text {ord }}$ according to the classification scheme introduced by [16] and [12]. To describe the different types of location problems we use a 5-position classification scheme Pos1/Pos2/Pos3/Pos4/Pos5, which allows us to indicate the number of new facilities (Pos1), the type of the problem as planar, network-based, discrete, etc. (Pos2), any assumption and restriction such as $w_{m}=1$ for all $m \in \mathcal{M}$, etc. (Pos3), the type of distance function such as $l_{p}$, general distance function $d$, etc. (Pos4), and the type of objective function (Pos5) (see [16] for further details).

The reader may note that problem $1 / \mathbb{R}^{n} / \bullet / \gamma_{B} / \sum_{\text {ord }}$ is somehow similar to the well-known Weber Problem, but it is more general because it includes as particular instances the Weber problem $\left(\lambda_{1}=\lambda_{2}=\ldots=\lambda_{n}=1\right)$, the $\alpha$-cent-dian problem $\left(\lambda_{1}=\ldots \lambda_{n-1}=1-\alpha\right.$ and $\left.\lambda_{n}=1\right)$ and the center problem $\left(\lambda_{1}=\ldots=\lambda_{n-1}=0\right.$ and $\lambda_{n}=1$ ) among others.

Example 1.1 Consider three demand points $a_{1}=(1,2), a_{2}=(3,5)$ and $a_{3}=(2,2)$ with weights $w_{1}=w_{2}=w_{3}=1$. Now choose $\lambda_{1}=\lambda_{2}=\lambda_{3}=1$ then we get $F(x)=$ $\sum_{i=1}^{3}\left\|x-a_{i}\right\|$, i.e. the Weber problem. For the second case choose $\lambda_{1}=\lambda_{2}=1 / 2$ and $\lambda_{3}=1$ then we get: $F(x)=1 / 2 \sum_{i=1}^{3}\left\|x-a_{i}\right\|+1 / 2 \max _{1 \leq i \leq 3}\left\|x-a_{i}\right\|$, i.e. the 1/2-centdian problem. Finally choose $\lambda_{1}=\lambda_{2}=0$ and $\lambda_{3}=1$ and we get: $F(x)=$ $\max _{1 \leq i \leq 3}\left\|x-a_{i}\right\|$, i.e. the center problem.

Also note that the objective function of this problem is region-wise defined and in general non convex if no additional hypotheses are assumed on the set $\Lambda$ (see [6] for further details).

Example 1.2 Consider two demand points $a_{1}=(0,0)$ and $a_{2}=(10,5), \lambda_{1}=100$ and $\lambda_{2}=1$ with $l_{1}$-norm and $w_{1}=w_{2}=1$. We obtain only two optimal solutions to Problem (1), lying in each demand point. Therefore the objective function is not convex since we have a nonconvex optimal solution set.

$$
\begin{aligned}
F\left(a_{1}\right) & =100 \times 0+1 \times 15=15 \\
F\left(a_{2}\right) & =100 \times 0+1 \times 15=15 \\
F\left(\frac{1}{2}\left(a_{1}+a_{2}\right)\right) & =100 \times 7.5+1 \times 7.5=757.5
\end{aligned}
$$




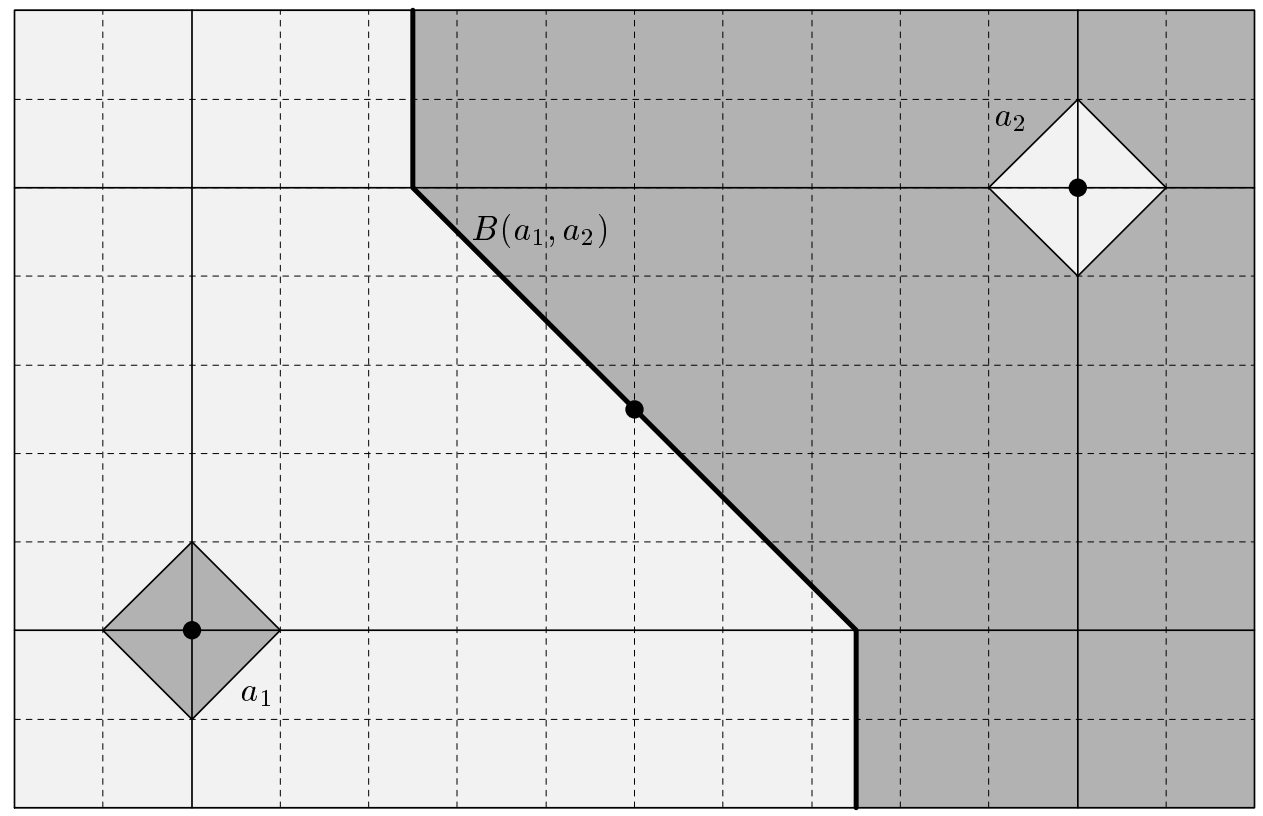

Figure 1: Illustration to Example 1.2

These two characteristics allow to model many different problems as we will show in the following.

The aforementioned paper by Puerto and Fernández [19] focuses only on developing the theoretical properties of this problem. Neither algorithms have been presented nor complexity aspects have been addressed. Exactly this will be the aim of this paper.

The outline of the paper is as follows: first the geometrical properties of (1) with polyhedral gauges are exploited. Then an efficient algorithm for the single facility case is given. The next section is devoted to extensions of Problem (1) to the multifacility case. After that the cases of restricted problems and general gauges are investigated and an approximation result is given. The paper ends with some conclusions and an out-view on future research.

\section{Geometrical Properties}

We are mainly interested in problems with polyhedral gauges. For this reason we will assume in the rest of the paper that $B \subseteq \mathbb{R}^{n}$ is a bounded polytope whose interior contains the zero and we denote the set of extreme points of $B$ by $\operatorname{Ext}(B)=\left\{e_{g}: g=\right.$ $1, \ldots, G\}$.

The polar set $B^{o}$ of $B$ is given by

$$
B^{o}=\left\{x \in \mathbb{R}^{n}:\langle x, p\rangle \leq 1 \quad \forall p \in B\right\}
$$

In the polyhedral case, $B^{o}$ is also a polytope, whose extreme points are $\left\{e_{g}^{o}: g=\right.$ $1,2, \ldots, G\}$, in $\mathbb{R}^{2}$, see [21] and [8]. 
The normal cone to $B$ at $x$ is given by

$$
N(B, x):=\left\{p \in \mathbb{R}^{n}:\langle p, y-x\rangle \leq 0 \quad \forall y \in B\right\}
$$

and the boundary of $B$ is denoted by $b d(B)$.

In this section we address some geometrical properties of the planar formulation of Problem (1) (denoted by $1 / \mathbb{R}^{2} / \bullet / \gamma_{B} / \sum_{\text {ord }}$ ) which give us specific insights in the considered model. In this case we define fundamental directions $d_{1}, \ldots, d_{G}$ as the halflines defined by 0 and $e_{1}, \ldots, e_{G}$. Further, we define $\Gamma_{g}$ as the cone generated by $d_{g}$ and $d_{g+1}$ (fundamental directions of $B$ ) where $d_{G+1}:=d_{1}$. Let $\pi=\left(p_{i}\right)_{i \in \mathcal{M}}$ be a family of elements of $\mathbb{R}^{2}$ such that $p_{i} \in B^{o}$ for each $i \in \mathcal{M}$ and let $\mathrm{C}_{\pi}=\bigcap_{i \in \mathcal{M}}\left(a_{i}+N\left(B^{o}, p_{i}\right)\right)$. A nonempty convex set $\mathrm{C}$ is called an elementary convex set if there exists a family $\pi$ such that $\mathrm{C}_{\pi}=\mathrm{C}$.

It should be noted that if the unit balls are polytopes we can obtain the elementary convex sets as intersection of cones generated by fundamental directions of these balls pointed at each demand point. Therefore each elementary convex set is a polyhedron whose vertices are called intersection points (see Figure 2). Finally, in the case of $\mathbb{R}^{2}$ there exists an upper bound of the number of elementary convex sets which is $O\left(M^{2} G^{2}\right)$. For further details see Durier and Michelot [8].

First of all, it should be noted that in Problem (1) although the objective function looks like the one of the Weber problem we do not have a unified linear representation of such a function in the whole space.

From the definition of the objective function, it is easy to see, that the representation may change every time $\gamma\left(x-a_{i}\right)-\gamma\left(x-a_{j}\right)$ becomes 0 for some $i, j \in\{1, \ldots, M\}$ with $i \neq j$. We will develop in this section a geometrical description of the sets where the representation of the objective function as a weighted sum stays unchanged.

Definition 2.1 The set $B_{\gamma}\left(a_{i}, a_{j}\right)$ consisting of points $\left\{x: w_{i} \gamma\left(x-a_{i}\right)=w_{j} \gamma(x-\right.$ $\left.\left.a_{j}\right), i \neq j\right\}$ is called bisector of $a_{i}$ and $a_{j}$ with respect to $\gamma$.

As an illustration of Definition 2.1 one can see in Figure 2 the bisector line for the points $(1,4)$ and $(5,1)$ with the rectangular norm.

Once these structures have been introduced, we can characterize their behavior. In order to do that, we use the concept of elementary convex sets introduced by Durier and Michelot [8], which in $\mathbb{R}^{2}$ can be seen as the subdivision of the plane made off the fundamental directions of the unit balls of all the existing facilities.

Proposition 2.1 The bisector of $a_{i}$ and $a_{j}$ is a set of points verifying a linear equation within each elementary convex set.

\section{Proof:}

In an elementary convex set $\gamma\left(x-a_{i}\right)$ and $\gamma\left(x-a_{j}\right)$ can be written as $l_{i}\left(x-a_{i}\right)$ and $l_{j}\left(x-a_{j}\right)$ respectively, where $l_{i}$ and $l_{j}$ are linear functions. Therefore, $\gamma\left(x-a_{i}\right)=$ $\gamma\left(x-a_{j}\right)$ is equivalent to $l_{i}\left(x-a_{i}\right)=l_{j}\left(x-a_{j}\right)$ and the result follows.

We will now give a more exact description of the complexity of a bisector when we restrict ourselves to the two dimensional space $\mathbb{R}^{2}$. 


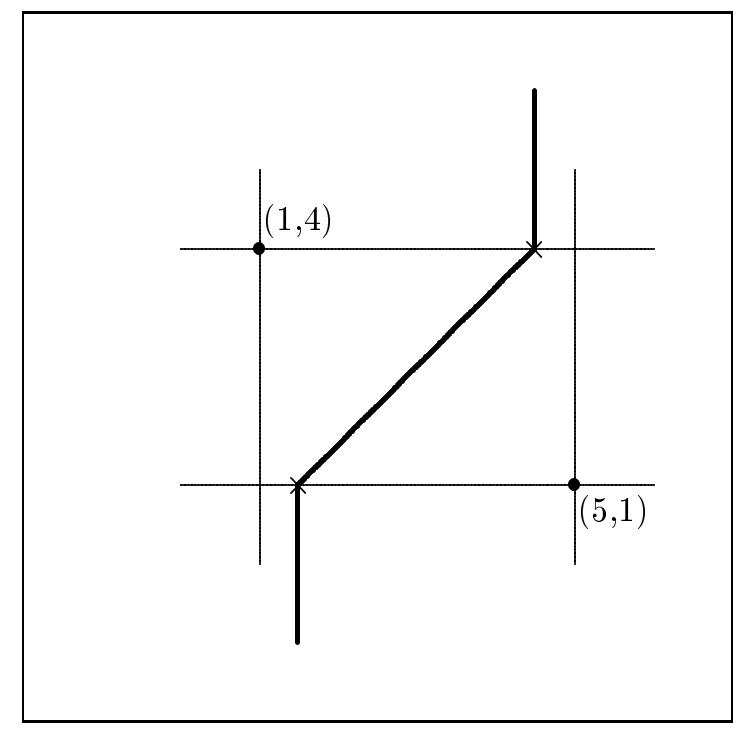

Figure 2: Bisector

Proposition 2.2 The bisector of $a_{i}$ and $a_{j}$ with respect to a polyhedral gauge $\gamma$ with $G$ extreme points has at most $O(G)$ different subsets defined by different linear equations.

\section{Proof:}

By Proposition 2.1 bisectors are set of points given by linear equations within e.c.s.. Therefore, the unique possible breakpoints may occur at the fundamental directions.

Let us denote by $L_{a_{i}}^{g}$ the fundamental direction starting at $a_{i}$ with direction $e_{g}$. On this halfline the function $\gamma\left(x-a_{i}\right)$ is linear with constant slope and $\gamma\left(x-a_{j}\right)$ is piecewise linear and convex. Therefore, the maximum number of zeros of $\gamma\left(x-a_{i}\right)-\gamma\left(x-a_{j}\right)$ when $x \in L_{a_{i}}^{g}$ is two. Hence, there are at most two breakpoints of the bisector of $a_{i}$ and $a_{j}$ on $L_{a_{i}}^{g}$.

Repeating this argument for any fundamental direction we obtain that an upper bound for the number of breakpoints is $4 G$.

This result implies that the number of different linear expressions defining any bisector is also linear in $G$, the number of fundamental directions. Remark that on some occasions bisector sets may have non empty interior, see for instance Figure 3, where we show the bisector set defined by the points $(0,0)$ and $(4,0)$ with the Tchebychev norm.

When at least two points are considered simultaneously the set of bisectors builds a subdivision of the plane (very similar to the well-known $k$-order Voronoi diagrams, see the book of Okabe et al. [17]). The cells of this subdivision will be called from now on ordered regions. We formally introduce this concept.

Definition 2.2 Given a permutation $\sigma$ on the set $\{1,2, \ldots, M\}$ the ordered region $O_{\sigma}$ 


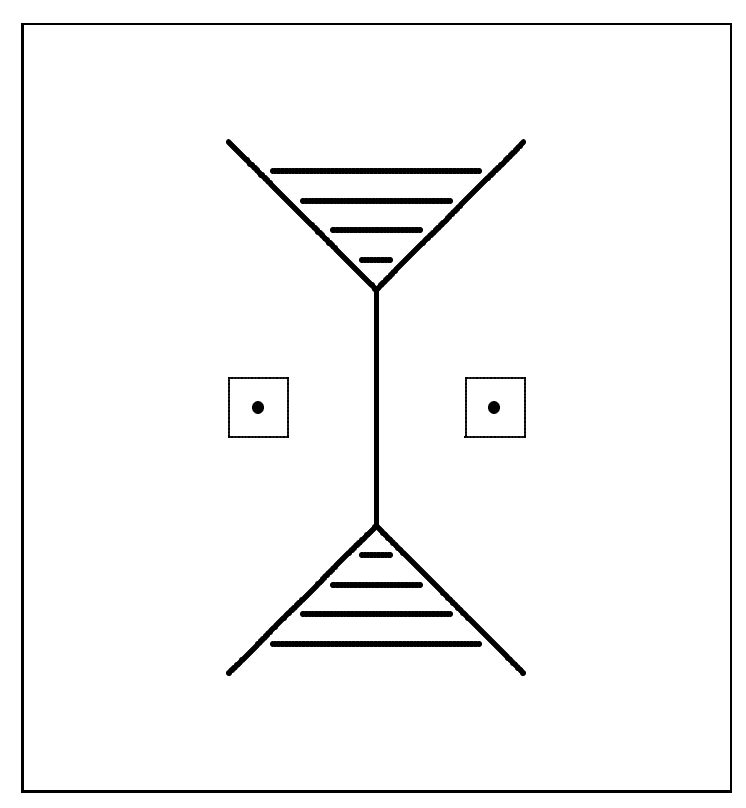

Figure 3: An example for a degenerated bisector

consists of the following set of points

$$
O_{\sigma}=\left\{x \in \mathbb{R}^{2}: \gamma\left(x-a_{\sigma_{1}}\right) \leq \ldots \leq \gamma\left(x-a_{\sigma_{M}}\right)\right\}
$$

Notice that these regions need not be convex sets, see Figure 4.

The importance of these regions is that in their intersection with e.c.s. the problem $1 / \mathbb{R}^{n} / \bullet / \gamma_{B} / \sum_{\text {ord }}$ behaves like a Weber problem, i.e the objective function has a unique linear representation. The intersections between ordered regions and e.c.s. are called following Puerto and Fernández [19] generalized elementary convex sets (g.e.c.s.). The ordered regions play a very important role in the algorithmic approach developed for solving the problem. In terms of bisectors, these regions are cells defined by at most $M-1$ bisectors of the set $A$.

However, the main disadvantage of dealing with these regions is their complexity. A naive analysis could lead to conclude that their number is $M$ ! which would make the problem intractable. Fortunately, we can obtain a polynomial bound which allows us to develop in the next section an efficient algorithm for solving Problem (1).

Theorem 2.1 An upper bound on the number of ordered regions is $O\left(M^{4} G^{2}\right)$.

\section{Proof:}

Given two bisectors with $O(G)$ linear pieces the maximum number of intersections is $O\left(G^{2}\right)$. The whole number of bisector of $M$ points is $\left(\begin{array}{c}M \\ 2\end{array}\right)$, then, the maximum number of intersections between them is $O\left(G^{2}\left(\begin{array}{c}M \\ 2 \\ 2\end{array}\right)\right)$. By Euler formula the number of intersections has the same complexity that the number of regions. Hence, an upper bound for the number of ordered regions is $O\left(M^{4} G^{2}\right)$.

A detailed analysis of this theorem shows that this bound is not too bad. Although, it is of order $M^{4} G^{2}$, it should be noted that the number of bisectors among the points in 


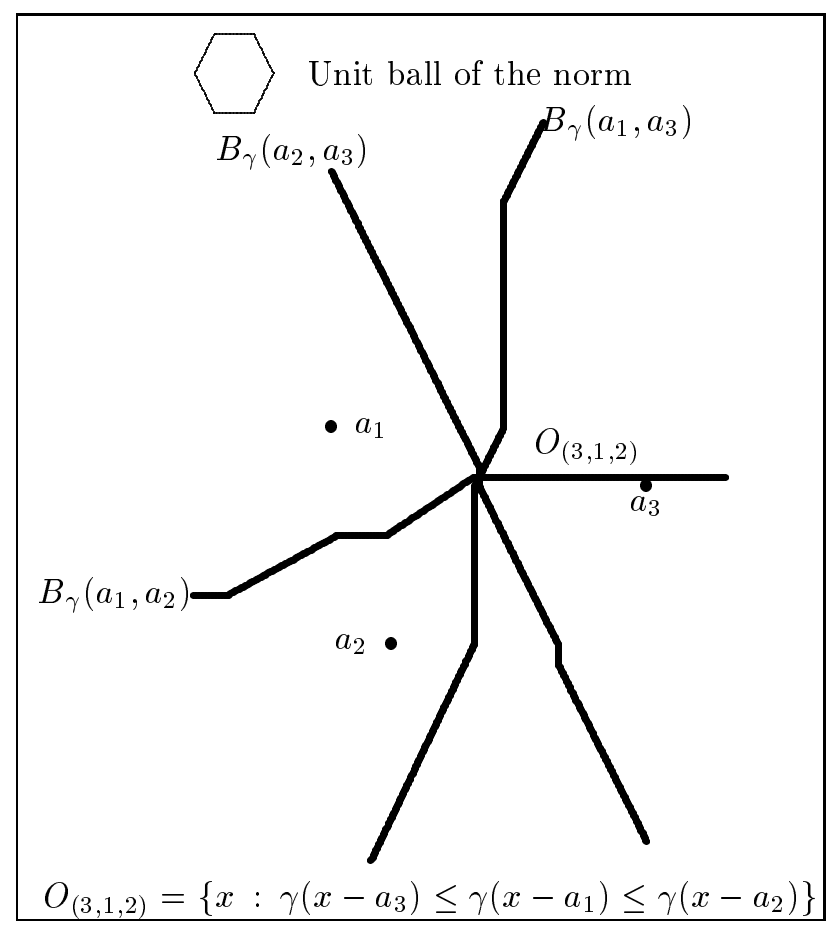

Figure 4: Ordered Regions

A is $\left(\begin{array}{c}M \\ 2\end{array}\right)$ which is order $M^{2}$. Therefore, even in the most favorable case of straight lines the number of regions in worst case analysis gives $O\left(\left(\begin{array}{c}M \\ 2\end{array}\right)^{2}\right)$ which is, in fact $O\left(M^{4}\right)$. Since our bisectors are polygonal with $G$ pieces this bound is rather tight.

Example 2.1 Figure 4 shows the ordered regions between the points $a_{1}=(0,11)$, $a_{2}=(3,0)$ and $a_{3}=(16,8)$ with the hexagonal norm whose set of extreme points is $\operatorname{Ext}(B)=\{(2,0),(1,2),(-1,2),(-2,0),(-1,-2),(1,-2)\}$. For instance, the region $O_{(3,2,1)}$ is the set of points

$$
\left\{x \in \mathbb{R}^{2}: \gamma\left(x-a_{3}\right) \leq \gamma\left(x-a_{2}\right) \leq \gamma\left(x-a_{1}\right)\right\}
$$

Finally, we quote for the sake of completeness a result stated in [19] which geometrically characterizes the solution set of the ordered Weber location problem: "The whole set of optimal solutions of Problem (1) always coincides with some generalized elementary convex sets". This is to say, the solution set coincides with the intersection of ordered regions with elementary convex sets [8].

\section{Single Facility Model}

Once we have introduced the main geometrical properties of this new model, we want to develop an algorithmic approach for solving the single facility ordered Weber problem. 
It should be noted that for the Weber's problem with polyhedral norms several algorithms have been proposed, see e.g. [5, 20, 21].

First of all, we state a well-known reformulation for $F(x)$ the proof of which can be found for example in Theorem 368 in [13].

Lemma 3.1 If the scalars in the set $\Lambda$ satisfy $\lambda_{1} \leq \ldots \leq \lambda_{M}$ then

$$
F(x)=\sum_{i=1}^{M} \lambda_{i} \gamma(x-A)_{(i)}=\max _{\sigma \in P(M)} \sum_{i=1}^{M} \lambda_{i} \gamma\left(x-a_{\sigma_{i}}\right)
$$

being $P(M)$ the set of permutations of $\{1, \ldots, M\}$.

Remark. This formulation can be interpreted as a worst-case approach with respect to all the possible weight arguments. From now on, we will consider that the lambdas satisfy $\lambda_{1} \leq \ldots \leq \lambda_{M}$.

Lemma 3.2 F is a convex function.

\section{Proof:}

By the previous lemma, $F(x)$ is the maximum of convex functions and is therefore convex.

Moreover, Puerto and Fernández [19] proved that the set of optimal solutions of Problem (1) always coincides with some generalized elementary convex sets. However, the large number of generalized elementary convex sets requires some kind of good enumeration scheme to derive an algorithm.

Since we restrict ourselves to polyhedral gauges a simple approach can be given. Within an ordered region $O_{\sigma}$, consider the following linear program:

$$
\begin{array}{lll}
\min & \sum_{i=1}^{M} \lambda_{i} z_{\sigma_{i}} \\
\text { s.t } & \left\langle e_{g}^{o}, x-a_{i}\right\rangle \leq z_{i} & e_{g}^{o} \in B^{o}, i=1,2, \ldots, M \\
& z_{\sigma_{i}} \leq z_{\sigma_{i+1}} & i=1,2, \ldots, M-1
\end{array}
$$

where $e_{g}^{o}$ are the fundamental directions of $B^{o}$.

Lemma 3.3 If an optimal solution $X^{*}$ of $P_{\sigma}$ is in $O_{\sigma}$ then $X^{*}$ is also an optimal solution to the ordered Weber problem constrained to $O_{\sigma}$.

\section{Proof:}

At an optimal point $X^{*}$ in $O_{\sigma}$ we have

$$
\left\langle e_{g}^{o}, X^{*}-a_{i}\right\rangle=z_{i} \quad, i=1,2, \ldots, M, \text { for some } g,
$$

which means that $z_{i}=\gamma\left(X^{*}-a_{i}\right)$ and the result follows.

Lemma 3.4 If an optimal solution $X^{*}$ of $P_{\sigma}$ is in $O_{\sigma^{\prime}} \neq O_{\sigma}$ then the optimal solution of the ordered Weber problem constrained to $O_{\sigma^{\prime}}$ is better than the optimal solution of the ordered Weber problem constrained to $O_{\sigma}$. 


\section{Proof:}

At an optimal point $X^{*}$ of $P_{\sigma}$ in $O_{\sigma^{\prime}}$ we have

$$
\left\langle e_{g}^{o}, X^{*}-a_{i}\right\rangle<z_{i} \quad \text { for all } g
$$

for at least one $i$. This means that we can decrease the objective function by moving from $O_{\sigma}$ to $O_{\sigma^{\prime}}$ and the result follows.

Based on Lemma 3.4 and the fact that the objective function is globally convex we develop a descent algorithm for this problem. For each ordered region we solve the problem as a linear program which geometrically means either finding the locally best solution in this ordered region or finding out that this region does not contain the global optimum by Lemma 3.4. In the former case two situations may occur. First, if the solution lies in the interior of the considered region (in $\mathbb{R}^{n}$ ) then by convexity this is the global optimum and secondly, if the solution is on the boundary we have to do a local search in the neighbourhood regions where this point belongs to.

\section{ALGORITHM 3.1.}

Step 1 Choose $x^{o}$ as an appropriate starting point. Initialize $\mathcal{L}:=\emptyset, y^{*}=x^{o}$.

Step 2 Look for the ordered region, $O_{\sigma^{\circ}}$ which $y^{*}$ belong to, where $\sigma^{\circ}$ determines the order.

Step 3 Solve the linear program $P_{\sigma^{0}}$. Let $u^{0}=\left(x_{1}^{0}, x_{2}^{0}, z_{\sigma}^{0}\right)$ be an optimal solution. If $x^{0}=\left(x_{1}^{0}, x_{2}^{0}\right) \notin O_{\sigma^{\circ}}$ then determine a new ordered region $O_{\sigma^{\circ}}$, where $x^{0}$ belongs to and go to Step 3.

Step 4 Let $y^{o}=\left(x_{1}^{0}, x_{2}^{0}\right)$.

Step 5 If $y^{o}$ belongs to the interior of $O_{\sigma^{\circ}}$ then set $y^{*}=y^{0}$ and go to Step 8.

Step 6 If $F\left(y^{o}\right) \neq F\left(y^{*}\right)$ then $\mathcal{L}:=\left\{\sigma^{0}\right\}$

Step 7 If there exist $i$ and $j$ verifying

$\gamma\left(y^{o}-a_{\sigma_{i}^{o}}\right)=\gamma\left(y^{o}-a_{\sigma_{j}^{o}}\right) \quad i<j \quad$ such that $\left(\sigma_{1}^{o}, \ldots, \sigma_{j}^{o}, \ldots, \sigma_{i}^{o}, \ldots, \sigma_{n}^{o}\right) \notin \mathcal{L}$

then do

a) $y^{*}:=y^{o}, \sigma^{o}:=\left(\sigma_{1}^{o}, \sigma_{2}^{o}, \ldots, \sigma_{j}^{o}, \ldots, \sigma_{i}^{o}, \ldots, \sigma_{M}^{o}\right)$

b) $\mathcal{L}:=\mathcal{L} \cup\left\{\sigma^{o}\right\}$

c) GO TO Step 3

else Go to Step 8 (Optimum found)

Step 8 Output $y^{*}$ 


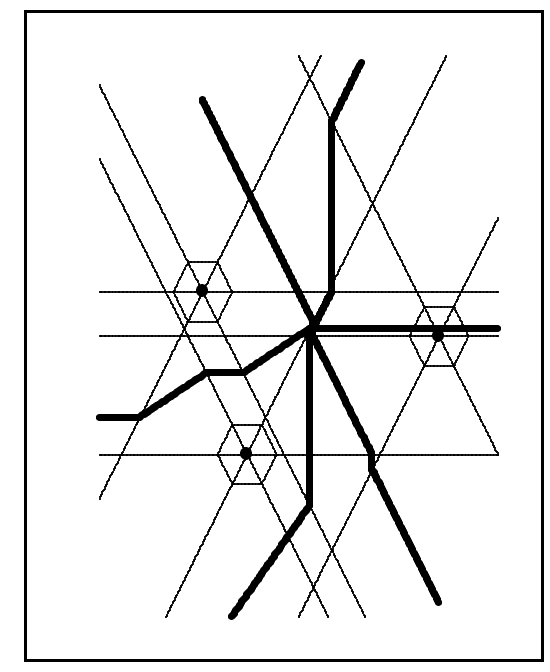

Figure 5: Illustration for Example 3.1

The above algorithm is efficient in the sense that it is polynomially bounded. Once the dimension of the problem is fixed, its complexity is dominated by the complexity of solving a linear program for each ordered region. Since the number of ordered regions is polynomially bounded and Megiddo's algorithm, [15], solves linear programs in fixed dimension in linear time, Algorithm 3.1 is linear in the number of cells.

Example 3.1 Consider the problem

$$
\min _{x \in \mathbb{R}^{2}} \gamma(x-A)_{(1)}+2 \gamma(x-A)_{(2)}+3 \gamma(x-A)_{(3)}
$$

where $A=\{(3,0),(0,11),(16,8)\}$ and $\gamma_{B}$ is the hexagonal polyhedral norm with $\operatorname{Ext}(B)=\{(2,0),(1,2),(-1,2),(-2,0),(-1,-2),(1,-2)\}$.

We show in Figure 5 the generalized elementary convex sets for this problem. Notice that the thick lines represent the bisectors for the points in $A$, while the thin ones are the fundamental directions of the norm. We solve the problem using Algorithm 3.1. Starting with $x^{o}=(0,11)$ we get the optimal solution in two iterations. In the first one, we get the point $x^{1}=(6.5,8)$ with objective value 26.25 . In the second iteration, we obtain $x^{2}=(7,8)$ with objective value 26 . This point can not be improved in its neighbourhood, therefore it is the optimal solution.

The iterations given by the algorithm for this example are depicted in Figure 6.

\section{Extension to the Multifacility Case}

A natural extension of the single facility model consists of considering the location of $N$ new facilities rather than only one. In this formulation the new facilities are chosen to provide service to all the existing facilities minimizing an ordered objective function. It should be noted that these ordered problems are of course harder to handle than the 
classical ones not considering ordered distances. Therefore, as no complexity results are known for the ordinary multifacility problem nothing can be said about the complexity of the ordered Weber problem. Needless to say that its resolution is even much more difficult than for single facility models.

Before formalizing the above problem, we should distinguish two different approaches that come from two different interpretations of the new facilities to be located. The first one assumes that the new facilities are not interchangeable, which means that they are of different importance for the existing facilities. The second one assigns the same importance to all new facilities. Here, we are only interested in the size of the distances, which means that we do not consider order among the new facilities and look for equity in the service, minimizing the largest distances.

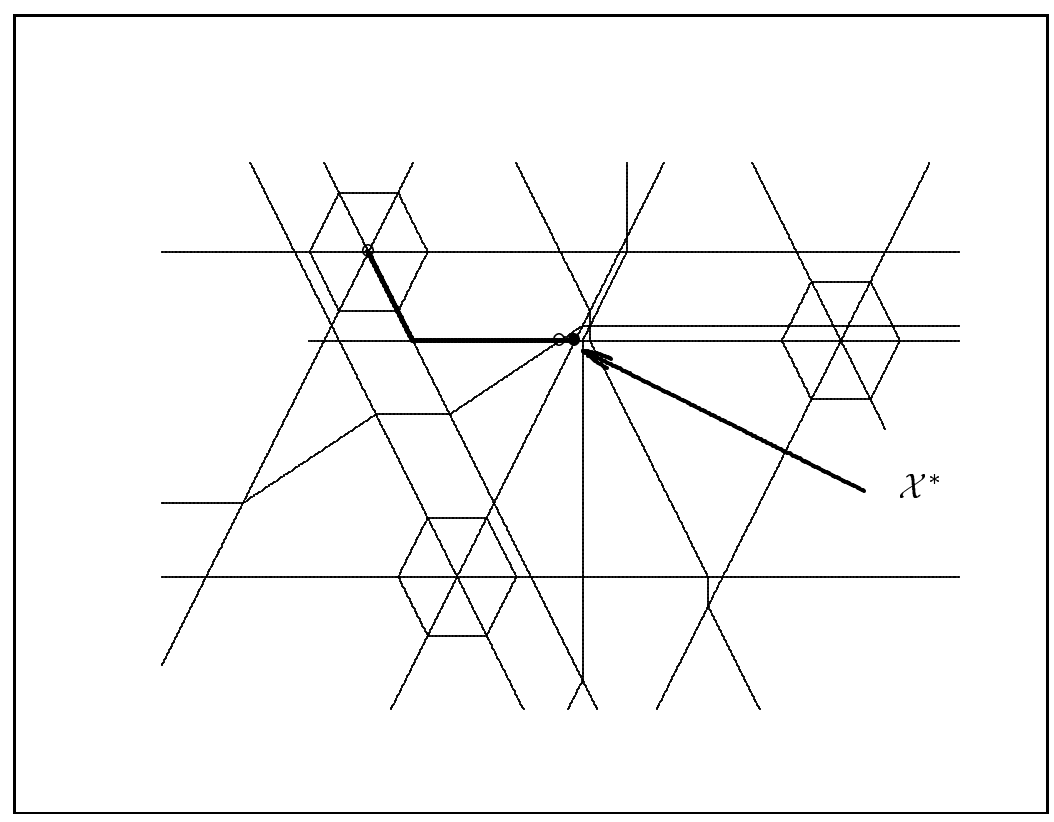

Figure 6: Optimal Solution

\subsection{The Non-Interchangeable Multifacility Model}

Let us consider a set of demand points $A=\left\{a_{1}, a_{2}, \ldots, a_{M}\right\}$. We want to locate $N$ new facilities $X=\left\{x_{1}, x_{2}, \ldots, x_{N}\right\}$ which minimize the following expression:

$$
F_{I}\left(x_{1}, x_{2}, \ldots, x_{N}\right)=\sum_{i=1}^{N} \sum_{j=1}^{M} \lambda_{i j} \gamma\left(x_{i}-A\right)_{(j)}+\sum_{k=1}^{N} \sum_{l=1}^{N} \mu_{k l} \gamma\left(x_{k}-x_{l}\right)
$$

where

$\lambda_{11} \leq \lambda_{12} \leq \ldots \leq \lambda_{1 M} \leq \lambda_{21} \leq \lambda_{22} \leq \ldots \leq \lambda_{2 M} \leq \ldots \leq \lambda_{N 1} \leq \lambda_{N 2} \leq \ldots \leq \lambda_{N M}$ 
$\mu_{k l} \geq 0$ for any $k=1, \ldots, N, l=1, \ldots, N$ and $\gamma\left(x_{i}-A\right)_{(j)}$ is the expression, which appears at the $j-t h$ position in the ordered list

$$
\mathcal{M}_{i}=\left\{w_{p} \gamma\left(x_{i}-a_{p}\right), p=1,2, \ldots, M\right\} \quad \text { for } i=1,2, \ldots, N .
$$

Remark that in this formulation we assign importance in increasing order of the index of the new facilities, i.e., $x_{j}$ is considered to be more important than $x_{i}$ whenever $i<j$. For this reason we say that this model has no-interchangeable facilities. With the same classification scheme [16] used for the single facility model, we will refer to this problem as $N / \mathbb{R}^{n} / \lambda_{\text {ord }} / \gamma_{B} / \sum_{\text {ord }}$.

In order to illustrate this approach we show an example which will serve as motivation for the following:

We consider a set of little towns and we want to locate one hospital and two emergency services (orthopedic surgery and cardiology). Our objective is to place the hospital and the emergency services such that the largest distance from the hospital to any town, the distances from any town to the emergency services and the distances from emergency services to the hospital are small. With these hypotheses we have to use a 3 -facility model, where the hospital has the greatest weight because it is the most important service and the two emergency services may have equal weights.

As in the single facility model we can prove that the objective function (3) is convex, which eases the analysis of the problem and the development of an efficient algorithm.

Proposition 4.1 The objective function $F_{I}$ is convex

\section{Proof:}

We know that

$$
\sum_{i=1}^{N} \sum_{j=1}^{M} \lambda_{i j} \gamma\left(x_{i}-A\right)_{(j)}=\sum_{i=1}^{N} \max _{\sigma^{i}} \sum_{j=1}^{N} \lambda_{i j} w_{\sigma_{j}^{i}} \gamma\left(x_{i}-a_{\sigma_{j}^{i}}\right)
$$

where $\sigma^{i}$ is a permutation of the set $\{1,2, \ldots, M\}$. Therefore, the first part of the objective function is a sum of maxima of convex functions analogous to Lemma 3.1. Hence, it is a convex function. On the other hand, the second term of the objective function $F_{I}$ is convex. Thus, $F_{I}$ is a convex function as a sum of convex functions.

The problem $N / \mathbb{R}^{n} / \lambda_{\text {ord }} / \gamma_{B} / \sum_{\text {ord }}$ can be transformed within the new ordered regions in the same way that we did for $1 / \mathbb{R}^{n} / \lambda_{\text {ord }} / \gamma_{B} / \sum_{\text {ord }}$. It should be noted that in $\mathbb{R}^{n}$ the subdivisions induced by the ordered regions of this problem are given as intersection of $N$ subdivisions. Each one of these $N$ subdivisions determines the ordered regions of each new facility.

Let $\sigma^{k}=\left(\sigma_{1}^{k}, \ldots, \sigma_{M}^{k}\right) k=1, \ldots, N$ be the permutations which give the order of the lists $\mathcal{M}_{k}$ introduced in (4). Consider the following linear program $\left(P_{\sigma}^{I}\right)$ :

$$
\min \sum_{k=1}^{N} \sum_{l=1}^{M} \lambda_{k l} z_{k \sigma_{l}^{k}}+\sum_{i=1}^{N} \sum_{j=1}^{N} \mu_{i j} y_{i j}
$$


s.t.

$$
\begin{array}{rl}
w_{l}\left\langle e_{g}^{o}, x_{k}-a_{l}\right\rangle \leq z_{k l} & e_{g}^{o} \in B^{o}, \quad k=1,2, \ldots, N, \quad l=1,2, \ldots, M \\
\left\langle e_{g}^{o}, x_{i}-x_{j}\right\rangle \leq y_{i j} & i=1,2, \ldots, N, \quad j=i+1, \ldots, M \\
z_{k \sigma_{l}^{k}} \leq z_{k \sigma_{l+1}^{k}} & k=1,2, \ldots, N, \quad l=1,2, \ldots, M-1
\end{array}
$$

Then, Algorithm 3.1 can easily be adapted to accommodate the multifacility case. Note than in contrary to that algorithm where we look for one point in $\mathbb{R}^{n}$ we now look for $N$ points in $\mathbb{R}^{n}$ or equivalently for one point in $\mathbb{R}^{n N}$. To do that, we only have to modify Step 1 by choosing $N$ starting points instead of one. In addition, we also have to consider that now the ordered regions are defined by different permutations, one from each list $\mathcal{M}_{i}$. Therefore, we have to replace the linear program $P_{\sigma}$ by $P_{\sigma}^{I}$ and to adapt its set of optimal solutions.

Since this algorithm is essentially the same that the one proposed for the single facility model, we can conclude that it is also polynomial bounded, hence applicable.

Example 4.1 Consider the two-facility problem.

$$
\begin{aligned}
& \min _{x_{1}, x_{2} \in \mathbb{R}^{2}} \quad 2.5 \gamma_{B}\left(x_{1}-A\right)_{(4)}+2 \gamma_{B}\left(x_{1}-A\right)_{(3)}+1.5 \gamma_{B}\left(x_{1}-A\right)_{(2)}+\gamma_{B}\left(x_{1}-A\right)_{(1)}+ \\
& +0.75 \gamma_{B}\left(x_{2}-A\right)_{(4)}+0.1 \gamma_{B}\left(x_{2}-A\right)_{(3)}+0.1 \gamma_{B}\left(x_{2}-A\right)_{(2)}+0.1 \gamma_{B}\left(x_{2}-A\right)_{(1)}+ \\
& +0.5 \gamma_{B}\left(x_{1}-x_{2}\right)
\end{aligned}
$$

where $A=\{(3,0),(0,11),(16,8),(-4,-7)\}$, and $\gamma_{B}$ is the hexagonal polyhedral norm, which we used in Example 3.1.

We obtain in the second iteration the optimal solution, with starting points $x_{1}^{o}=$ $(0,11)$ and $x_{2}^{o}=(16,8)$. The optimal solution is $(2.75,5.5)$ and $(3.125,5.875)$. The elementary convex set and the optimal solution can be seen in Figure 7 .

\subsection{The Indistinguishable Multifacility Model}

The multifacility model that we are considering now differs from the previous one in the sense that the new facilities are similar from the users point of view. Therefore, the new facilities have no different importance with respect to the existing ones. On the contrary, the weight given to each one of these new facilities depends only on the size of the distances.

Using the same notation as in Section 4.1, the objective function of this model is:

$$
F_{I I}\left(x_{1}, x_{2}, \ldots, x_{N}\right)=\sum_{j=1}^{N M} \lambda_{j} \gamma(x-A)_{(j)}+\sum_{i=1}^{N} \sum_{j=1}^{N} \mu_{i j} \gamma\left(x_{i}-x_{j}\right)
$$

where

$$
\lambda_{1} \leq \lambda_{2} \leq \ldots \leq \lambda_{N M}
$$

and $\gamma(x-A)_{(j)}$ is the expression which appears at the $j-t h$ position in the following ordered list

$$
\mathcal{M}_{I I}=\left\{w_{p} \gamma\left(x_{k}-a_{p}\right), k=1,2, \ldots, N, \quad p=1,2, \ldots, M\right\} .
$$




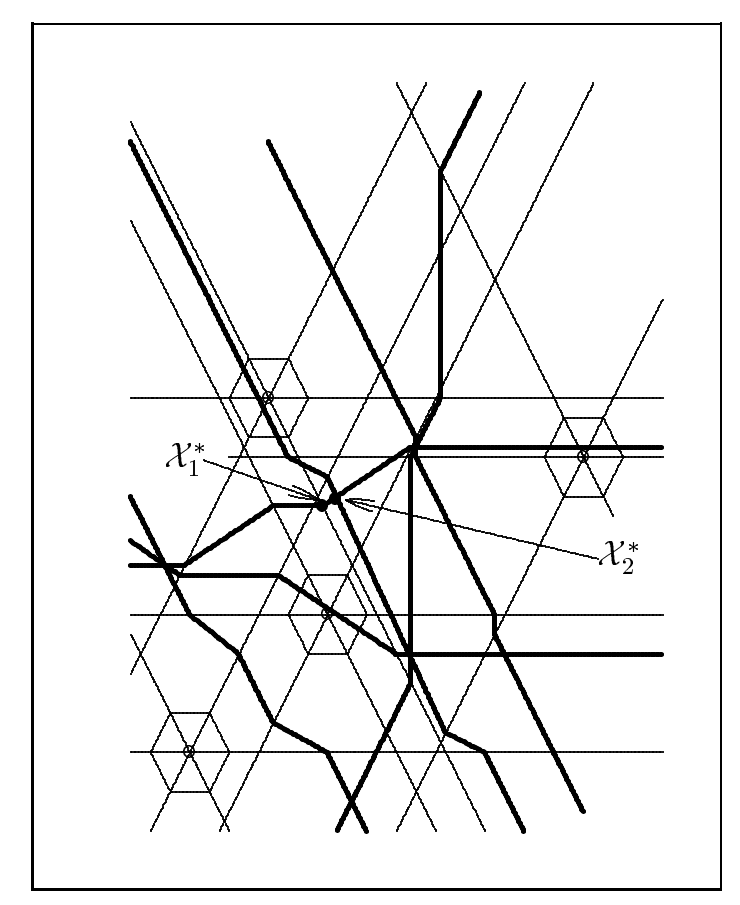

Figure 7: Illustration for Example 4.1.

According to the classification scheme this problem is written as $N / \mathbb{R}^{n} / \bullet / \gamma_{B} / \sum_{\text {ord }}$. Also this model is motivated by a hypothetical real situation:

Consider a University, with 10 departments and 3 assistant services to be located (computer center, library and lecture hall). Any department need to have access to all 3 services. In addition, it is also assumed that the computer center, the library and the lecture hall have to communicate.

Our objective is to minimize the sum of all distances. Besides, we want to prevent services from being too far away from each others. With these hypotheses this situation can be formulated as a 3-facility indistinguishable model where we want to locate three facilities depending only on the size of the distances.

Proposition 4.2 The objective function $F_{I I}$ is convex

The proof is analogous to the one given for Proposition 4.1.

Using again the same strategy that we have already used for the non-interchangeable multifacility model, the problem $N / \mathbb{R}^{n} / \bullet / \gamma_{B} / \sum_{\text {ord }}$ can be solved using an adaptation of Algorithm 3.1.

Let $\sigma$ be a permutation of $\{1, \ldots, M N\}$ where $\sigma_{(k-1) M+j}$ gives the position of $w_{j} \gamma\left(x_{k}-a_{j}\right)$ in $\mathcal{M}_{I I}$.

Consider the following linear programming problem $\left(P_{\sigma}^{I I}\right)$ :

$$
\min \sum_{k=1}^{N} \sum_{l=1}^{M} \lambda_{\sigma_{k l}} z_{\sigma_{(k-1) M+l}}+\sum_{p=1}^{N} \sum_{q=1}^{N} \mu_{p q} y_{p q}
$$


s.t.

$$
\begin{array}{rl}
w_{l}\left\langle e_{g}, x_{k}-a_{l}\right\rangle \leq z_{(k-1) M+l} & e_{g} \in B^{o} \quad k=1,2, \ldots, N \quad l=1,2, \ldots, M \\
\left\langle e_{g}, x_{p}-x_{q}\right\rangle \leq y_{p q} & p=1,2, \ldots, N \quad q=1, \ldots, N \\
z_{\sigma_{(k-1) M+l}} \leq z_{\sigma_{(k-1)+l+1}} & k=1,2, \ldots, N \quad l=1,2, \ldots, M-1
\end{array}
$$

Once we replace $P_{\sigma}^{I}$ by $P_{\sigma}^{I I}$ we can easily adapt the algorithmic approach showed for the previous model in Section 4.1. Hence, the same conclusions that we obtained for $N / \mathbb{R}^{n} / \lambda_{\text {ord }} / \gamma_{B} / \sum_{\text {ord }}$ are applicable to $N / \mathbb{R}^{n} / \bullet / \gamma_{B} / \sum_{\text {ord }}$.

\section{Extensions}

\subsection{Restricted Case}

In the last years an area within location theory which has attained considerable attention is the restricted facility location problems, see for instance, Brady and Rosenthal [3], Drezner [7], Karkazis [14], Aneja and Palar [1]. Also the work of Francis et al. [9] in which a contour line approach is given is related to this topic and Hamacher and Nickel [10] and Nickel [16], describe a concept for solving restricted location problem, besides they gave a combinatorial algorithm to restricted Weber problem.

In this section we study the problems considered in the previous sections when forbidden regions are allowed to exist.

It should be noted that the unrestricted single and multifacility case that we have studied before can be extended easily to the restricted case. We will assume that there is a forbidden region $\mathcal{R}$ containing all the optimal solutions of the unrestricted problem. This hypothesis is necessary because otherwise we can get the optimal solution by solving the unrestricted problem. Besides, if the number of forbidden regions is greater than one, to obtain the optimal solution of the restricted problem, we have only to consider the region which contains the optimal solution of the unrestricted problem.

First, we extend these results to the case where the forbidden region is a general convex set. In order to locate the new facilities, we use the following result

Theorem 5.1 For $1 / \mathbb{R}^{2} / \mathcal{R} / \gamma_{B} / \sum_{\text {ord }}$ with polyhedral gauges there is always an optimal solution on the 0 -dimensional intersections between the boundary of $\mathcal{R}$, the fundamental directions and the bisector lines.

\section{Proof:}

Using the same arguments as in Theorem 2.4.5 in [16], it follows that the optimal solutions of the restricted ordered facility location problem is on the boundary of the forbidden region. Moreover, the objective function is linear in each generalized elementary convex set, see Lemma 3.4, and the proof follows analogous to [16].

As an immediate consequence of the Theorem 5.1 we state the following algorithm for solving the single facility problems with a forbidden region, $\mathcal{R}$.

\section{ALGORITHM 5.1.}


Step 1 Compute the fundamental directions and bisector lines for all existing facilities.

Step 2 Determine $\left\{y_{1}, y_{2}, \ldots, y_{k}\right\}$ the intersection points between fundamental directions or bisector lines and the boundary of the forbidden region, $\mathcal{R}$.

Step 3 Compute $x_{\mathcal{R}}^{*} \in \operatorname{argmin}\left\{f\left(y_{1}\right), f\left(y_{2}\right), \ldots, f\left(y_{k}\right)\right\}\left(x_{\mathcal{R}}^{*}\right.$ is an optimal solution to the restricted location problem).

Step 4 The set of optimal solutions is $\left\{x: f(x)=f\left(x_{\mathcal{R}}^{*}\right)\right\}$ intersected with the boundary of $\mathcal{R}$.

For the particular case of polyhedral forbidden regions we can get better results. Let $\mathcal{R}$ be a polyhedral forbidden region, $\left\{s_{1}, s_{2}, \ldots, s_{k}\right\}$ the set of facets of $\mathcal{R}$ and $A=\left\{a_{1}, \ldots, a_{M}\right\}$ the set of existing facilities.

\section{ALGORITHM 5.2.}

Step 1 Let $p:=1, \mathcal{L}:=\emptyset$ and let $y^{*}$ be an arbitrary feasible solution.

Step 2 Consider the hyperplane $\mathcal{T}_{p}$ defined by the facet $s_{p}$ of $\mathcal{R}$ and choose $x^{o}$ belonging to the relative interior of $s_{p}$. Let $\mathcal{T} \leq_{p}$ be the halfplane which does not contain $\mathcal{R}$ and let $x^{*}=x^{o}$.

Step 3 Determine the ordered region $O_{\sigma^{\circ}}$ where $x^{o}$ belongs to, and the permutation $\sigma^{o}$ which determines this region. region on Solve the following linear program

$$
\begin{array}{lll}
\min & \sum_{i=1}^{M} \lambda_{i} z_{\sigma_{i}^{o}} \\
\text { s.t } & \left\langle b_{g}, x-a_{i}\right\rangle \leq z_{i} \quad e_{g} \in B^{o}, i=1,2, \ldots, N \\
& z_{\sigma_{i}^{o}} \leq z_{\sigma_{i+1}^{o}} & i=1,2, \ldots, N-1 \\
& x \in \mathcal{T} \leq{ }_{p}
\end{array}
$$

Step 5 Let $u^{o}=\left(x^{o}, z_{\sigma}^{o}\right)$ be an optimal solution of $P_{\mathcal{T} \leq_{p}}$. If $x^{o} \notin O_{\sigma^{o}}$ then go to Step 3 .

Step 6 If $x^{0}$ belongs to the interior of $O_{\sigma^{\circ}}$ then let $x^{*}=x^{o}$ and go to Step 9

Step 7 If $F\left(x^{o}\right) \neq F\left(x^{*}\right)$ then $\mathcal{L}:=\emptyset$

Step 8 If there exist $i$ and $j$ verifying

$$
\gamma\left(x^{o}-a_{\sigma_{j}^{o}}\right)=\gamma\left(x^{o}-a_{\sigma_{i}^{o}}\right) \quad i<j \quad \text { such that }\left(\sigma_{1}^{o}, \ldots, \sigma_{j}^{o}, \ldots, \sigma_{i}^{o}, \ldots, \sigma_{n}^{o}\right) \notin \mathcal{L}
$$

Then do
a) $x^{*}:=x^{o}, \sigma^{o}:=\left(\sigma_{1}^{o}, \sigma_{2}^{o}, \ldots, \sigma_{j}^{o}, \ldots, \sigma_{i}^{o}, \ldots, \sigma_{n}^{o}\right)$
b) $\mathcal{L}:=\mathcal{L} \cup\left\{\sigma^{o}\right\}$
c) GO TO Step 4 .

Step 9 Do 

a) If $F\left(x^{*}\right)<F\left(y^{*}\right)$ then $y^{*}:=x^{*}$
b) $p:=p+1$.
d) If $p<k$ GO TO Step 2, otherwise the optimal solution is $y^{*}$.

Notice that this algorithm can be used to solve problems with convex forbidden regions not necessarily polyhedral. In order to do so we only have to approximate these regions by polyhedral ones. Since this approximation can be done with arbitrary precision using for instance the sandwich approximation of Burkard et al. in [4], we can get good approximations to the optimal solutions of the original problems.

\subsection{Non polyhedral case}

In the previous sections we only have considered polyhedral norms. We will use these results to develop a general scheme for solving the considered problems under general gauges (non necessarily polyhedral).

We show that the optimal solutions of these problems can be arbitrarily approximated by sequence of optimal solutions of problems with polyhedral gauges converging under the Haussdorf metric to the considered non-polyhedral one.

Although in this section, we only consider the objective function of the single facility case, $\mathrm{F}(\mathrm{x})$, all the results can be extended in an easy way to the multifacility cases.

Let $B$ be a unit ball of the gauge $\gamma_{B}(\cdot),\left\{B_{n}\right\}_{n \in \mathbb{N}}$ an increasing sequence of polyhedra included in $B$ and $\left\{B^{n}\right\}_{n \in \mathbb{N}}$ a decreasing sequence of polyhedra including $B$, that is,

$$
B_{n} \subset B_{n+1} \subset B \subset B^{n+1} \subset B^{n} \quad \text { for all } n=1,2, \ldots
$$

Let $\gamma_{B_{n}}(\cdot)$ and $\gamma_{B^{n}}(\cdot)$ be the gauges whose unit balls are $B_{n}$ and $B^{n}$ respectively.

Proposition 5.1 If $B_{n} \subset B \subset B^{n}$ we have that

$$
\gamma_{B_{n}}(x) \geq \gamma_{B}(x) \geq \gamma_{B^{n}}(x) \quad \forall x, \in \mathbb{R}^{n}
$$

The proof follows directly from the definition of gauges.

Recall that given two compact sets $A, B$ the Haussdorf distance between $A$ and $B$ is

$$
d_{H}(A, B)=\max \left(\max _{x \in A} d_{2}(x, B), \max _{y \in B} d_{2}(A, y)\right)
$$

where $d_{2}(x, B)=\min _{y \in B} d_{2}(x, y)$ being $d_{2}$ the Euclidean distance.

Proposition 5.2 Let $K$ be a compact set. If $B_{n}$ converges to $B$ and $B^{n}$ converges to $B$ under the Haussdorf metric then for all $\varepsilon>0$ there exists $n_{0}$ such that for all $n \geq n_{0}$

$$
\begin{aligned}
& \max _{x \in K}\left|F_{n}(x)-F(x)\right|<\varepsilon \\
& \max _{x \in K}\left|F^{n}(x)-F(x)\right|<\varepsilon
\end{aligned}
$$

being $F_{n}(x):=\sum_{i=1}^{M} \lambda_{i} \gamma_{B_{n}}(x-A)_{(i)}$ and $F^{n}(x):=\sum_{i=1}^{M} \lambda_{i} \gamma_{B^{n}}(x-A)_{(i)}$. 


\section{Proof:}

We only prove the first inequality. The second one follows analogously.

Since $B_{n}$ converges to $B$ under the Haussdorf metric verifying $B_{n} \subset B_{n+1}$ for all $n$, and $K$ is a compact set then given $\varepsilon>0$ there exists $n_{a}$ for all $a \in A$ such that if $n>n_{A}:=\max _{a \in A} n_{a}$ then $\left|\gamma_{B}(x-a)-\gamma_{B_{n}}(x-a)\right|<\frac{\varepsilon}{\sum_{i=1}^{M} w_{i} \sum_{i=1}^{M} \lambda_{i}} \quad \forall x \in K$.

By continuity we have that for any $i, j$ and any $x \in K$ verifying that $w_{i} \gamma_{B}\left(x-a_{i}\right)<$ $w_{j} \gamma_{B}\left(x-a_{j}\right)$ there exists $n_{0}$ such that for all $n>n_{0}$

$$
w_{i} \gamma_{B_{n}}\left(x-a_{i}\right)<w_{j} \gamma_{B_{n}}\left(x-a_{j}\right)
$$

On the other hand, if there exists $k, l$ and $x \in K$ such that $w_{k} \gamma_{B}\left(x-a_{k}\right)=$ $w_{l} \gamma_{B}\left(x-a_{l}\right)$ then there also exists $n_{0}$ and a permutation $\sigma^{n_{0}}$ such that for all $n>n_{0}$ it holds: 1) $w_{\sigma_{k}^{n_{0}}} \gamma_{B_{n}}\left(x-a_{\sigma_{k}^{n_{0}}}\right)=\gamma_{B_{n}}(x-A)_{(k)}$, and 2) $w_{\sigma_{k}^{n_{0}}} \gamma_{B}\left(x-a_{\sigma_{k}^{n_{0}}}\right)=\gamma_{B}(x-A)_{(k)}$. Hence, we have for any $x \in K$ and $n>\max \left\{n_{A}, n_{0}\right\}$ that

$$
\begin{gathered}
\gamma_{B}(x-A)_{(k)}=w_{\sigma_{k}^{n_{0}}} \gamma_{B}\left(x-a_{\sigma_{k}^{n_{0}}}\right) \\
\gamma_{B_{n}}(x-A)_{(k)}=w_{\sigma_{k}^{n_{0}}} \gamma_{B_{n}}\left(x-a_{\sigma_{k}^{n_{0}}}\right) .
\end{gathered}
$$

Therefore for any $x \in K$ and $n>\max \left\{n_{A}, n_{0}\right\}$ we obtain that

$$
\left|F_{n}(x)-F(x)\right|=\sum_{i=1}^{M} \lambda_{i}\left|\gamma_{B}(x-A)_{(i)}-\gamma_{B_{n}}(x-A)_{(i)}\right|=\sum_{i=1}^{M} \lambda_{i} w_{\sigma_{i}^{n_{0}}}\left|\gamma_{B}\left(x-a_{\sigma_{i}^{n_{0}}}\right)-\gamma_{B_{n}}\left(x-a_{\sigma_{i}^{n_{0}}}\right)\right|<\varepsilon
$$

Corollary 5.1 i) If $B_{n}$ converges to $B$ under the Haussdorf metric, then $F_{n}(x)$ converges to $F(x)$, besides the sequence $\left\{F_{n}(x)\right\}_{n \in \mathbb{N}}$ is decreasing.

ii) If $B^{n}$ converges to $B$ under the Haussdorf metric, then $F^{n}(x)$ converges to $F(x)$, besides the sequence $\left\{F^{n}(x)\right\}_{n \in \mathbb{N}}$ is increasing.

In the following, we use another kind of convergence, called epi-convergence see Definition 1.9 in the book of Attouch [2]. Let $\left\{g ; g^{\nu}, \nu=1, \ldots\right\}$ be a collection of extended-values functions. We say that $g^{\nu}$ epi-converges to $g$ if for all $x$,

$$
\begin{aligned}
& \inf _{x^{\nu} \rightarrow x} \liminf _{\nu \rightarrow \infty} g^{\nu}\left(x^{\nu}\right) \geq g(x) \\
& \inf _{x^{\nu} \rightarrow x} \limsup _{\nu \rightarrow \infty} g^{\nu}\left(x^{\nu}\right) \leq g(x)
\end{aligned}
$$

where the infima are with respect to all subsequences converging to $x$. The epiconvergence is very important because it establishes a relationship between the convergence of functionals and the convergence of the sequence of their minima. Further details can be found in the book of Attouch [2].

Our next result states the theoretical convergence of the proposed scheme.

Theorem $5.2 \quad$ i) Let $\left\{x_{n}\right\}_{n \in \mathbb{N}}$ be a sequence such that $x_{n} \in \arg \min F_{n}(x)$ then any accumulation point of $\left\{x_{n}\right\}_{n \in \mathbb{N}}$ belongs to $\arg \min F$. 
ii) Let $\left\{x^{n}\right\}_{n \in \mathbb{N}}$ be a sequence such that $x^{n} \in \arg \min F^{n}(x)$ then any accumulation point of $\left\{x_{n}\right\}_{n \in \mathbb{N}}$ belongs to $\arg \min F$.

\section{Proof:}

We only prove the first part, because the proof of the second one is built on the same pattern using Proposition 2.41. in [2] instead of Proposition 2.48.

First of all, since the sequence $\left\{F_{n}\right\}_{n \in \mathbb{N}}$ is a decreasing sequence applying Theorem 2.46 in [2] we obtain that the sequence $\left\{F_{n}(x)\right\}_{n \in \mathbb{N}}$ is epi-convergent.

In addition, we get from Proposition 2.48 in [2] that

$$
\lim _{n \rightarrow \infty} \inf _{x \in \mathbb{R}^{2}} F_{n}(x)=\inf _{x \in \mathbb{R}^{2}} \lim _{n \rightarrow \infty} F_{n}(x)=\inf _{x \in \mathbb{R}^{2}} F(x)
$$

Since $\mathbb{R}^{n}$ is a first countable space and $\left\{F_{n}\right\}_{n \in \mathbb{N}}$ is epi-convergent, we get from Theorem 2.12 in [2] that any accumulation point of the sequence $\left\{x_{n}\right\}_{n \in \mathbb{N}}$ is an optimal solution of the problem with objective function $F$.

\subsection{Conclusions}

In this paper we have developed efficient algorithm for the ordered Weber problems introduced by [19] for the case of polyhedral gauges. Also extensions to the multifacility case have been developed. In addition a discussion of the non polyhedral case and the case with forbidden regions has been presented. For the planar case discussion of the geometrical properties of the generalized elementary convex sets has been given. Therefore, we have provided a new flexible tool for modelling and solving a broad range of location problems.

Further research includes the analysis of multicriteria formulation of these problems as well as a detailed study about ordered Weber problems with some negative weights.

\section{References}

[1] Aneja, Y. P. And Parlar, M. "Algorithms for Weber facility location in the presence of forbidden regions and/or barriers to travel". Transactions Science, 28, 70-76, 1994.

[2] Aттоuch, H. "Variational convergence for functions and operators". Pitman Advanced Publishing Program, 1984.

[3] Brady, S.D. and Rosenthal, R.E. "Interactive computer graphical minimax location of multiple facilities with general constraints" . AIIE Transactions, 15, 242-252, 1983.

[4] Burkard, R.E., Hamacher H.W. and Günter R. " Sandwich Approximation of Univariate Convex functions with an application to separable convex programming" . Naval Research Logistics, 38, 911-924, 1991. 
[5] Carrizosa, E. And Puerto, J. "A discretizing algorithm for location problems". Europeam Journal of Operational Research, 80, 166-174, 1995.

[6] Crouseix, J.P. And Kebbour, R. "On the convexity of some simple functions of ordered samples". JORBEL, 36 11-25 (1996).

[7] Drezner, Z. "Constrained location problems in the plane and on a sphere". AIIE Transportation, 12, 300-304, 1983.

[8] Durier, R. And Michelot, C. "Geometrical properties of Fermat-Weber Problem". European Journal of Operational Research, 20, 332-343, 1985.

[9] Francis, R. L., Hamacher, H. W., Lee, C. Y. and Yeralan, S. "On automating robotic assembly workplace planning" . IIE Transactions, 47-59, 1994.

[10] Hamacher, H. W. AND Nickel S. "Combinatorial algorithms for some 1facility median problems in the plane" . European Journal of Operational Research, 79, 340-351, 1994.

[11] Hamacher, H. W. AND NiCKEL S. "Restricted planar location problems and applications". Naval Research Logistics, 42, 967-992, 1995.

[12] Hamacher, H. W. And Nickel, S. "Multicriteria planar location problems." European Journal of Operational Research, 94, 66-86, 1996.

[13] Hardy G.H., J.E. Littlewood and G. Polya "Inequalities" Cambridge University Press. 1952.

[14] KarKaZIS, J. " The general unweighted problem of locating obnoxious facilities on the plane". Belgian Journal of Operations Research, Statistics and Computer Science, 28, 3-49, 1988.

[15] Megiddo, N. "Linear programming in linear time when the dimension is fixed". Journal of the Assotiation for Computing Machinery, 31, 114-127, 1984.

[16] Nickel S. “Discretization of planar location problems". Verlag Shaker, 1995.

[17] Okabe A., Barry B. and Sugihara K. "Spatial Tessellations Concepts and Applications of Voronoi Diagrams". John Wiley \& Sons , 1992.

[18] Plastria, F. "Continuous location problems: research, results and questions". Facility Location: A Survey of Applications and Methods, (Z. Drezner, Ed.). Springer-Verlag, 85-127, 1995.

[19] Puerto, J. And Fernández F.R. "The symmetrical single facility location problem". Prepublicación de la Facultad de Matemáticas n. 34. Universidad de Sevilla, 1995.

[20] Puerto J. "Lecturas en Teoría de Localización". Universidad de Sevilla. Secretariado de Publicaciones. (Ed. Justo Puerto), 1996. 
[21] Ward J.E. AND Wendell R.E. "Using block norms for location modelling". Operations Research. 33, 1074-1090, 1985. 